\title{
Acute and sustained effects of early administration of inhaled nitric oxide to children with acute respiratory distress syndrome*
}

\author{
José R. Fioretto, MD, PhD; Marcos A. de Moraes, MD; Rossano C. Bonatto, MD, PhD; \\ Sandra M. Q. Ricchetti, MD; Mário F. Carpi, MD
}

Objective: To determine the acute and sustained effects of early inhaled nitric oxide on some oxygenation indexes and ventilator settings and to compare inhaled nitric oxide administration and conventional therapy on mortality rate, length of stay in intensive care, and duration of mechanical ventilation in children with acute respiratory distress syndrome.

Design: Observational study.

Setting: Pediatric intensive care unit at a university-affiliated hospital.

Patients: Children with acute respiratory distress syndrome, aged between 1 month and 12 yrs.

Interventions: Two groups were studied: an inhaled nitric oxide group (iNOG, $n=18$ ) composed of patients prospectively enrolled from November 2000 to November 2002, and a conventional therapy group (CTG, $n=21$ ) consisting of historical control patients admitted from August 1998 to August 2000.

Measurements and Main Results: Therapy with inhaled nitric oxide was introduced as early as $1.5 \mathrm{hrs}$ after acute respiratory distress syndrome diagnosis with acute improvements in $\mathrm{PaO}_{2} /$ $\mathrm{F}_{2}$ ratio $(83.7 \%)$ and oxygenation index (46.7\%). Study groups were of similar ages, gender, primary diagnoses, pediatric risk of mortality score, and mean airway pressure. $\mathrm{PaO}_{2} / \mathrm{FIO}_{2}$ ratio was lower (CTG, $116.9 \pm 34.5$; iNOG, $62.5 \pm 12.8, p<.0001)$ and oxygenation index higher (CTG, 15.2 [range, 7.2-32.2]; iNOG, 24.3 [range, 16.3-70.4], $p<.0001$ ) in the iNOG. Prolonged treatment was associated with improved oxygenation, so that $\mathrm{FiO}_{2}$ and peak inspiratory pressure could be quickly and significantly reduced. Mortality rate for inhaled nitric oxide-patients was lower (CTG, ten of $21,47.6 \%$; iNOG, three of $18,16.6 \%, p<.001)$. There was no difference in intensive care stay (CTG, 10 days [range, 2-49]; iNOG, 12 [range, 6-26], $p>.05$ ) or duration of mechanical ventilation (TCG, 9 days [range, 2-47]; iNOG, 10 [range, 4-25], p $>$.05).

Conclusions: Early treatment with inhaled nitric oxide causes acute and sustained improvement in oxygenation, with earlier reduction of ventilator settings, which might contribute to reduce the mortality rate in children with acute respiratory distress syndrome. Length of stay in intensive care and duration of mechanical ventilation are not changed. Prospective trials of inhaled nitric oxide early in the setting of acute lung injury in children are needed. (Pediatr Crit Care Med 2004; 5:469-474)

KEY WORDS: inhaled nitric oxide; acute respiratory distress syndrome; children; mechanical ventilation; respiratory failure; intensive care ince its first description (1), acute respiratory distress syndrome (ARDS) is still a therapeutic challenge in pediatric intensive care, and it has been associated with high mortality rate, despite better understanding of its pathophysiology and recent therapeutic advances (2).

\footnotetext{
*See also p. 496.

From the Pediatric Intensive Care Unit, Department of Pediatrics, São Paulo State University-Unesp, Botucatu Medical School, São Paulo, Brazil.

Supported, in part, by FAPESP, process number 2001/04971-3.

Address requests for reprints to: José Roberto Fioretto, MD, PhD, UNESP, Faculdade de Medicina de Botucatu, Departamento de Pediatria, 18.618-970, Botucatu, São Paulo, Brasil. E-mail: jrf@fmb.unesp.br

Copyright ( 2004 by the Society of Critical Care Medicine and the World Federation of Pediatric Intensive and Critical Care Societies
}

DOI: 10.1097/01.PCC.0000137986.83738.D7
The local effects of inhaled nitric oxide (iNO) on oxygenation, inflammation, pulmonary hypertension, edema, and capillary permeability may account for its use in ARDS. Rossaint et al. (3) first demonstrated in adult ARDS patients that iNO decreases intrapulmonary shunting and improves arterial oxygenation. Abman et al. (4), in 1994, described the beneficial effects of iNO on oxygenation, pulmonary hypertension, and cardiac index in children with ARDS. Afterward, many studies performed on adults (5-9) and children (10-15), although confirming those effects, were not able to demonstrate a sustained response to iNO therapy. However, Dobyns et al. (16) observed sustained response to iNO vs. placebo therapy in subgroups of pediatric patients (oxygenation index $\geq 25$ and immunocompromised group). These authors explained that iNO therapy did not sustain oxygenation improvement in all patients because they were enrolled in the study in the later stages of the disease.

The hypothesis that the response to iNO therapy depends on its time of introduction had already been stated by others $(17,18)$, supporting the idea that early iNO treatment may be more effective (19, $20)$. Recently (21), starting iNO administration as early as 12 hrs after ARDS diagnosis, we demonstrated acute and sustained response of oxygenation indexes.

In 2002, The Cochrane Library (22) published a systematic review on the effects of iNO in acute hypoxemic respiratory failure in children and adults. Five randomized controlled trails were evaluated, assessing 535 patients. The review concluded that iNO may be useful only as a rescue treatment in first 24 hrs of the disease. However, it is worth mentioning that of these five studies, only one was performed in children (16). Therefore, 
the question about the potential role of iNO therapy in ARDS is still open and remains to be defined, mainly in children.

The aims of this study were a) to determine the acute and sustained effects of early iNO on some oxygenation indexes and ventilator settings, to analyze the weaning process, and to assess the safety of nitric oxide inhalation; and b) to compare iNO administration plus conventional therapy with just conventional therapy on mortality rate, length of stay in intensive care, and duration of mechanical ventilation (MV) in children with ARDS.

\section{METHODS}

This study was approved by the Human Research and Ethics Committee of the University Hospital of Botucatu Medical School. Written informed consent was obtained from the parents or guardians of each child before enrollment.

Patients. Children with ARDS, aged between 1 month and $12 \mathrm{yrs}$, and admitted to the Pediatric Intensive Care Unit (PICU), were considered potential subjects for this study. ARDS was defined according to the AmericanEuropean Consensus Conference published in 1994 (23).

To assess the acute and sustained effects of iNO, children admitted prospectively from November 2000 to November 2002 were selected for the iNO group (iNOG, $n=18$ ) only if they showed $<88 \%$ arterial oxygen saturation despite a positive end-expiratory pressure (PEEP) $\geq 10 \mathrm{~cm} \mathrm{H}_{2} \mathrm{O}$ and $\mathrm{FiO}_{2} \geq 0.6$.

A historical control group was used for comparison (conventional therapy group, or CTG, $n=21$ ). These children, admitted from August 1998 to August 2000, were part of a series of patients treated with conventional therapy only, published in 2001 (24), to assess pediatric ARDS incidence, gas exchange, and mortality rate in our PICU. The same inclusion criteria were used to select control patients. Therefore, from 24 patients who originally composed the historical group, three were excluded (two died and one survived). Control group demographic data are presented in Table 1.

Our aim was also to study ARDS patients without chronic cardiac or pulmonary diseases; therefore, patients with these diseases were excluded.

The patients were monitored according to standard PICU protocol. All the children had a radial artery catheter for continuous monitoring of systolic and diastolic pressures and mean arterial pressure (MAP) and for blood gas sampling. A central venous catheter was used to obtain other biochemical values to calculate Pediatric Risk of Mortality (PRISM) III (25) score and to assess coexisting multiple organ system failure. Lung function status

Table 1. Conventional therapy group demographic data

\begin{tabular}{|c|c|c|c|c|c|c|}
\hline $\begin{array}{l}\text { Patient } \\
\text { No. }\end{array}$ & $\begin{array}{l}\text { Age, Months/ } \\
\text { Gender }\end{array}$ & $\begin{array}{l}\text { ARDS } \\
\text { Etiology }\end{array}$ & $\begin{array}{l}\text { Other } \\
\text { MOSF }\end{array}$ & $\begin{array}{c}\text { PRISM } \\
\text { (Mortality } \\
\text { Risk, \%) }\end{array}$ & $\begin{array}{l}\text { Inotropic } \\
\text { Support }\end{array}$ & Outcome \\
\hline 1 & $8 / \mathrm{F}$ & Septic shock & $\mathrm{CV}$ & $33(51)$ & DA, Dob, NE & Survived \\
\hline 2 & 7/M & Septic shock & $\mathrm{CV}, \mathrm{C}$ & $29(37)$ & Dob & Survived \\
\hline 3 & 96/M & Trauma & CV, C, K, L & $25(46)$ & DA, Dob & Died \\
\hline 4 & $3 / \mathrm{F}$ & Septic shock & CV, C, K, L & $27(53)$ & DA, Dob & Died \\
\hline 5 & $12 / \mathrm{M}$ & Septic shock & $\mathrm{CV}, \mathrm{C}, \mathrm{K}$ & $22(47)$ & DA, Dob, NE & Died \\
\hline 6 & $72 / \mathrm{F}$ & Septic shock & $\mathrm{CV}, \mathrm{C}, \mathrm{K}, \mathrm{N}$ & $27(48)$ & Dob & Died \\
\hline 7 & $2 / \mathrm{M}$ & Septic shock & $\mathrm{CV}, \mathrm{C}, \mathrm{R}$ & $32(51)$ & DA, Dob & Died \\
\hline 8 & $3 / \mathrm{F}$ & Septic shock & $\mathrm{CV}, \mathrm{C}, \mathrm{K}, \mathrm{L}, \mathrm{N}$ & $41(53)$ & DA, Dob & Died \\
\hline 9 & 4/M & Septic shock & $\mathrm{CV}, \mathrm{C}$ & $18(35)$ & Dob & Died \\
\hline 10 & $13 / \mathrm{F}$ & Septic shock & $\mathrm{CV}$ & $17(11)$ & Dob & Survived \\
\hline 11 & $113 / \mathrm{M}$ & Septic shock & & $14(12)$ & & Survived \\
\hline 12 & 4/M & Pneumonia & & $15(16)$ & & Survived \\
\hline 13 & $5 / \mathrm{M}$ & Pneumonia & & $19(34)$ & & Died \\
\hline 14 & $12 / \mathrm{F}$ & Septic shock & $\mathrm{CV}$ & $17(22)$ & Dob & Survived \\
\hline 15 & 6 & Pneumonia & - & $21(19)$ & & Survived \\
\hline 16 & $108 / \mathrm{M}$ & Septic shock & $\mathrm{CV}, \mathrm{K}, \mathrm{L}$ & $23(48)$ & Dob & Died \\
\hline 17 & 2/M & Septic shock & $\mathrm{CV}, \mathrm{K}$ & $25(59)$ & DA, Dob & Died \\
\hline 18 & $44 / \mathrm{F}$ & Pneumonia & $\mathrm{CV}, \mathrm{K}, \mathrm{N}$ & $17(11)$ & Dob & Survived \\
\hline 19 & $16 / \mathrm{F}$ & Pneumonia & & $16(17)$ & & Survived \\
\hline 20 & $1 / \mathrm{M}$ & Pneumonia & & $24(37)$ & & Survived \\
\hline 21 & $3 / \mathrm{F}$ & Pneumonia & & $18(32)$ & & Survived \\
\hline
\end{tabular}

ARDS, acute respiratory distress syndrome; MOSF, multiple organ system failure; PRISM, pediatric risk of mortality; F, female; $\mathrm{M}$, male; $\mathrm{CV}$, cardiovascular; C, coagulopathy; K, kidney; L, liver; DA, dopamine; Dob, dobutamine; NE, norepinephrine.

was assessed by oxygenation index (OI, mean airway pressure $\times \mathrm{FIO}_{2} \times 100 / \mathrm{PaO}_{2} ; \mathrm{cm}$ $\mathrm{H}_{2} \mathrm{O} / \mathrm{mm} \mathrm{Hg}$ ) and the $\mathrm{PaO}_{2} / \mathrm{FIO}_{2}$ ratio. OI was used both as a measure of oxygenation and as an indicator of mechanical ventilatory support aggressiveness.

Diagnosis of multiple organ system failure was based on the criteria proposed by Wilkinson et al. (26). Sepsis and septic shock were defined according to the American College of Chest Physicians/Society of Critical Care Medicine Consensus Conference (27).

Conventional Therapy. Initial ventilatory management was performed with time-cycled pressure-limited ventilators. PEEP was gradually increased to recruit lung volume and to attain open alveoli, while avoiding clinical and radiographic signs of lung hyperinflation. Peak inspiratory pressure (PIP) was limited to $\leq 35 \mathrm{~cm} \mathrm{H} \mathrm{H}_{2} \mathrm{O}$, permitting hypercapnia if necessary and accepting arterial saturation of oxygen between $88 \%$ and $90 \%$. The median respiratory rate was 30 (range, 25-45) per minute. The choice of ventilator was in accordance with the ventilation protocol established by the PICU, depending on the child's weight $(<10 \mathrm{~kg}$, Sechrist IV-100B, Sechrist Industries, Anaheim, CA; $>10$ kg, Inter 5, Intermed, São Paulo, Brazil). There were no modifications in MV style of practice throughout the study; permissive hypercapnia and permissive hypoxia were in use throughout both study periods, and no new mechanical ventilators were acquired. Therefore, iNOG and historical controls were both submitted to the same MV protocol. Another variable that could interfere with results is the use of cuffed tubes; tracheal tubes with cuff were used to treat children $>8 \mathrm{yrs}$ old, and leak around the tube was not measured.

Routine ARDS management procedure included treatment of the underlying diseases and sedation with continuous intravenous infusion of midazolam and/or fentanyl. The patients were paralyzed by continuous intravenous infusion of atracurium when necessary. Optionally, prone positioning was used as part of conventional treatment (28), and no patients from both groups received surfactant or steroids. Hemodynamic support included the optimization of intravascular fluid volume, guided by central venous pressure monitoring and administration of catecholamines.

Inhaled Nitric Oxide Administration. Inhaled nitric oxide (NO) administration followed the guidelines and techniques previously described $(21,29,30)$. Briefly, NO blended with nitrogen was obtained from $20-\mathrm{L}$ tanks connected to a pressure regulator (AGA Medical S.A., São Paulo, Brazil). The concentration in the tanks was certified by the suppliers as 300 parts per million (ppm) of nitric oxide in nitrogen. The NO was continuously delivered to the patients via flowmeter, directly into the inspiratory limb of the ventilator circuit, distal to humidifier from a point 30 $\mathrm{cm}$ distal to the child's tracheal tube. Inhaled nitric oxide and nitric dioxide $\left(\mathrm{NO}_{2}\right)$ concentrations were measured using an electrochemical sensor (J P Moryia Ind \& Com Ltda, São Paulo, Brazil) from samples of circuit gas obtained as close as possible to the tracheal tube via Y-piece. The $\mathrm{NO} / \mathrm{NO}_{2}$ electrochemical sensor gas analyzer was calibrated before use ev- 
ery day. Audiovisual alarms were calibrated at a dose of $1 \mathrm{ppm}$ above the iNO administered dose and at a maximum level of 3 ppm $\mathrm{NO}_{2}$ concentration. The delivery system was flushed thoroughly before use.

Inhaled Nitric Oxide Administration Protocol. Baseline measurements (time zero; $\mathrm{T}_{0}$ ) were made at steady-state pressure control ventilation immediately before starting iNO administration. The conventional therapy and ventilator settings were set and not changed during the 4-hr dose-response test. Inhaled NO was administered at a dose-response test of 20 ppm for 30 mins under the previously mentioned ventilator settings. Respiratory and hemodynamic measurements were then performed $\left(\mathrm{T}_{30 \text { mins }}\right)$. Regardless of the response, concentration was reduced to $10 \mathrm{ppm}$ and after a further 30 mins to $5 \mathrm{ppm}$. This latter dose was maintained for a further $3 \mathrm{hrs}$ to complete the 4-hr trial ( $\left.\mathrm{T}_{4 \mathrm{hrs}}\right)$. Measurements were taken at the end of each period. Positive response was defined as an increase in $\mathrm{PaO}_{2} /$ $\mathrm{FIO}_{2}$ ratio of 10 torr (31) above the baseline value, with 5 ppm dose at $\mathrm{T}_{4}$ hrs. If the patient did not respond, a new trial was performed with starting dose of 40 ppm for 30 mins and then 10 ppm for 30 mins and 5 ppm for 3 hrs. If the patient did not respond to this second trial, a trial with a fixed dose of $20 \mathrm{ppm}$ for 4 hrs was performed every day. Based on a positive response, iNO was continued at $5 \mathrm{ppm}$ until arterial saturation of oxygen $\geq 88 \%$ with $\mathrm{FIO}_{2}<0.6$ was achieved. The iNO therapy was then gradually withdrawn with decreases of 1 $\mathrm{ppm} / \mathrm{hr}$ over $6-12 \mathrm{hrs}$. If withdrawal caused a decrease in $\mathrm{PaO}_{2}$, requiring an increase of $\mathrm{FIO}_{2}$ by $\geq 20 \%$, iNO was reset to the previous level. The aim of this protocol was to maintain the lowest dose of iNO associated with an improvement in oxygenation. Daily mean iNO dose and the $\mathrm{FIO}_{2}$ and PIP levels were assessed from the end of the 4-hr dose-response test to the end of that day $(\mathrm{d} 0)$ and over the following days (d1, d2, d3...).

Methemoglobin concentration was measured immediately before and at each arterial blood gas analysis after the beginning of iNO therapy.

Statistical Analysis. Study groups were compared for patient age, gender, $\mathrm{PaO}_{2} / \mathrm{FIO}_{2}$ ratio, $\mathrm{OI}, \mathrm{MAP}, \mathrm{PaCO}_{2}$, multiple organ system failure diagnosis, catecholamine use, and PRISM III score. We also compared intensive care length of stay, duration of MV, ventilator settings (PIP, $\mathrm{FIO}_{2}, \mathrm{PEEP}$, and mean airway pressure) during treatment period, and mortality rate. Student's $t$-test was used to compare variables with normal distribution and Mann-Whitney test when nonnormal distribution occurred. Goodman's test was used to analyze gender and survival per group.

Friedman's repeated measures of variance test was used to compare $\mathrm{PaO}_{2} / \mathrm{FIO}_{2}$ ratio, OI, $\mathrm{PaCO}_{2}$, heart rate, and MAP at each moment of the 4-hr dose-response test. Student's $t$-test and Mann-Whitney test were used to compare ventilator settings and OI over the entire pe- riod of $\mathrm{iNO}$ use. $\mathrm{FiO}_{2}$ values between groups were compared using the profile analysis technique for two independent groups, considering four moments of evaluation; PIP values were compared using repeated measurements of variance test with two factors (groups and moments). Normally and nonnormally distributed data were expressed as mean \pm SD and median (ranges), respectively. Differences were considered significant at $p<0.05$.

\section{RESULTS}

Inhaled Nitric Oxide Therapy. Inhaled nitric oxide therapy was introduced early. The median duration between the time of ARDS diagnosis and initiation of iNO therapy was 1.5 hrs (range, 1-96), and patients had received MV for 1-216 hrs (median, 24.5 hrs) before enrollment.

iNOG-Acute Response to iNO Therapy. Table 2 shows that immediately before iNO therapy, the patients had marked oxygenation impairment demonstrated by the mean of the $\mathrm{PaO}_{2} / \mathrm{FIO}_{2}$ ratio $(70.08 \pm 24.1)$ and by the median of the OI of 29.95 (range, 19-75). All but one patient had a positive response at the end of $\mathrm{T}_{4}$ hrs. This child showed hemodynamic instability due to septic shock, being treated with dobutamine. Once stability was achieved, the patient became responsive and was included in the protocol. The mean percentage improvement in $\mathrm{PaO}_{2} / \mathrm{FIO}_{2}$ ratio and $\mathrm{OI}$ from baseline was $83.7 \%$ and $46.7 \%$, respectively. During the 4-hr dose-response test, whereas heart rate values decreased, MAP and $\mathrm{PaCO}_{2}$ did not show any significant variation. Partial arterial pressure of carbon dioxide values as high as 101.1 torr were observed using the permissive hypercapnia approach.

iNOG-Sustained Response to iNO Therapy. Figure 1 shows OI throughout the treatment period; iNO therapy pro-

Table 2. Acute Effect of Inhaled Nitric Oxide (iNO) on Oxygenation Indexes, Gas Exchange, and Hemodynamic Variables During the 4-hr Dose-Response Test

\begin{tabular}{lccr}
\hline \multirow{3}{*}{ Variable } & \multicolumn{3}{c}{ Test Times $(\mathrm{n}=18)$} \\
\cline { 2 - 4 } \multicolumn{1}{c}{$\mathrm{T}_{0}$} & $\mathrm{~T}_{30 \text { mins }}$ & \multicolumn{1}{c}{$\mathrm{T}_{4 \text { hrs }}$} \\
\hline $\mathrm{PaO}_{2} / \mathrm{FIO}_{2}$ & $70.08 \pm 24.1$ & $110.22 \pm 34.7^{a}$ & $128.72 \pm 50.6^{a}$ \\
$\mathrm{OI}_{\mathrm{cm} \mathrm{H}} \mathrm{O} / \mathrm{mm} \mathrm{Hg}$ & $29.95(19-75)$ & $18.55^{a}(10.1-44.6)$ & $15.95^{a, b}(5.2-32)$ \\
$\mathrm{HR}$, beats/min & $150 \pm 14.65$ & $145 \pm 15^{c}$ & $143 \pm 12^{c}$ \\
$\mathrm{MAP}, \mathrm{mm} \mathrm{Hg}$ & $59.56 \pm 10.86$ & $58.17 \pm 9.56$ & $57.94 \pm 8.34$ \\
$\mathrm{PaCO}_{2}$, torr & $55.15(20.7-92.2)$ & $53(22.5-85.2)$ & $50.3(17.5-101.1)$ \\
\hline
\end{tabular}

$\mathrm{T}_{0}$, data from baseline, immediately before iNO administration; $\mathrm{T}_{30 \text { mins }}$, data at 30 mins; $\mathrm{T}_{4}$ hrs, data at $4 \mathrm{hrs}$; OI, oxygenation index (mean airway pressure $\times \mathrm{FIO}_{2} \times 100 / \mathrm{PaO}_{2}$ ); HR, heart rate; MAP, mean arterial pressure.

${ }^{a} p<.001$ compared with $\mathrm{T}_{0} ;{ }^{b} p<.001$ compared with $\mathrm{T}_{30 \text { mins }} ;{ }^{c} p<.01$ compared with $\mathrm{T}_{0}$, Friedman test. Data are expressed as mean \pm SD and median (range). 


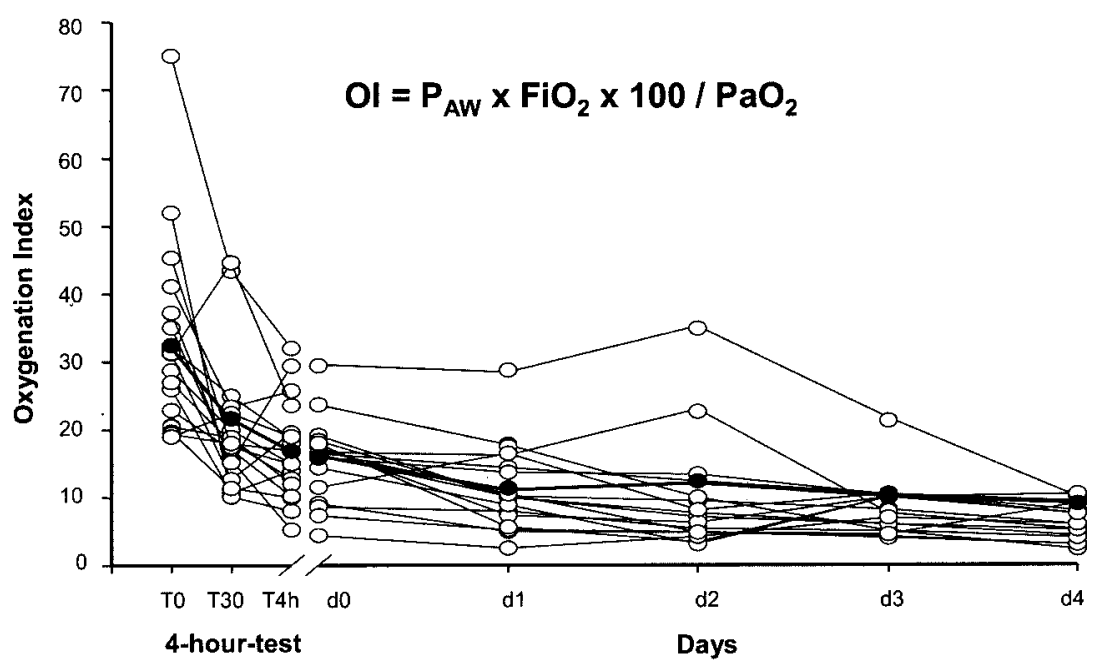

Figure 1. Evolution of oxygenation index $(O I)$ during the 4-hr dose-response test and prolonged inhaled nitric oxide (iNO) therapy for each patient, and the mean values (filled circles). Sustained improvement could be seen in all patients. Two patients developed "rebound" during weaning process. $T 0$, baseline values; T30, at 30 mins with $20 \mathrm{ppm}$ dose of iNO; T4h, end of the test with $5 \mathrm{ppm}$ dose of iNO; $d 0$, period from the end of dose-response test to the end of the day of the beginning of iNO therapy; $d 1-d 4$, the days of treatment; $P_{A W}$, mean airway pressure.

Table 3. Ventilator Settings During Inhaled Nitric Oxide (iNO) Treatment

\begin{tabular}{lcccc}
\hline & \multicolumn{4}{c}{ Day of Treatment } \\
\cline { 2 - 5 } \multicolumn{1}{c}{ Variable } & $\mathrm{D}_{0}$ & $\mathrm{D}_{1}$ & $\mathrm{D}_{2}$ & $\mathrm{D}_{3}$ \\
\hline $\mathrm{FIO}_{2}$ & $0.91 \pm 0.16$ & $0.66 \pm 0.17^{a}$ & $0.55 \pm 0.19^{a, b}$ & $0.51 \pm 0.07^{a, b}$ \\
$\mathrm{PIP}, \mathrm{cm} \mathrm{H} \mathrm{H}_{2} \mathrm{O}$ & $30(25-35)$ & $28^{a}(22-35)$ & $23.5^{a, b}(17-35)$ & $24^{a, b}(20-30)$ \\
\hline
\end{tabular}

$\mathrm{D}_{0}$, period from the end of dose-response test to the end of the day of the beginning of iNO therapy; $\mathrm{D}_{1}, \mathrm{D}_{2}, \mathrm{D}_{3}$, the days of treatment; $\mathrm{FIO}_{2}$, fraction of inspired oxygen; PIP, peak inspiratory pressure.

${ }^{a} p<.001$ compared with $\mathrm{D}_{0} ;{ }^{b} p<.001$ compared with $\mathrm{D}_{1}, t$ and Mann-Whitney tests. Data are expressed as mean $\pm \mathrm{SD}$ and median (range).

Table 4. Demographic and Clinical Characteristics of Patient Populations

\begin{tabular}{lccc}
\hline & $\begin{array}{c}\text { CTG } \\
(\mathrm{n}=21)\end{array}$ & $\begin{array}{c}\text { iNOG } \\
(\mathrm{n}=18)\end{array}$ & $p$ Value \\
\hline Age in months, median (range) & $7(1-113)$ & $12.5(1-132)$ & $>.05$ \\
Gender & 11 & 9 & $>.05$ \\
$\quad$ No. of males & 10 & 9 & \\
$\quad$ No. of females & $13(61.9)$ & $10(55.6)$ & \\
Primary diagnosis & $7(33.3)$ & $6(33.3)$ & \\
$\quad$ Sepsis/septic shock (\%) & $1(4.8)$ & $2(11.1)$ & $>.05$ \\
Pneumonia (\%) & $14(66.7)$ & $11(61.1)$ & $>.05$ \\
$\quad$ Trauma (\%) & $14(66.7)$ & $11(61.1)$ & $>.05$ \\
MOSF (\%) & $22.83 \pm 6.9$ & $20.56 \pm 4.8$ & \\
Catecholamines (\%) & & & \\
PRISM III score (\%) & & & \\
\hline
\end{tabular}

CTG, conventional therapy group; iNOG, inhaled nitric oxide group; MOSF, multiple organ system failure; PRISM, pediatric risk of mortality.

Tests: $t$-test, Mann-Whitney, and Goodman.

no statistical difference for intensive care stay (CTG, 10 days [range, 2-49]; iNOG, 12 [range, 6-26], $p>.05$ ) and duration of MV (CTG, 9 days [range, 2-47]; iNOG, 10 [range, 4-25], $p>.05$ ) between the groups.
$(5-7,9,33)$. For sustained response, however, there are a few reports on oxygenation index behavior over time in pediatric ARDS patients. One might expect that acute positive response could be sustained during the entire iNO therapy; this has been very difficult to demonstrate ( 6 , $7,12,13)$. While studying adult patients and starting iNO administration within 3 days of ARDS diagnosis, Dellinger et al. (33) observed an improvement in oxygenation index over the first 4 days. Michael et al. (7) observed no sustained response after $24 \mathrm{hrs}$ in patients in whom iNO therapy was started up to 25 days after ARDS diagnosis. These authors stated that the lack of response after $24 \mathrm{hrs}$ might be because the same mechanisms account for the oxygenation improvement with iNO or conventional therapy and that iNO may only bring them into play earlier. However, Michael et al. (7) included patients with severe disease who were not responding to standard therapy. In children, Dobyns et al. (16) only observed sustained iNO response over 72 hrs in immunocompromised and OI $\geq 25$ patients. The authors explained that oxygenation improvement was not sustained in all patients because they were included at later stages of the disease.

It was our aim to demonstrate that early iNO administration, in optimally ventilated patients with appropriate PEEP levels, can have a sustained response; this could reduce mortality rate, length of PICU stay, and MV duration.

Different from previously mentioned reports, we introduced iNO as part of our therapeutic approach as soon as possible after ARDS diagnosis. In addition to an acute positive response, we observed sustained improvement in oxygenation. We also demonstrated an early decrease in the ventilator settings that are associated with high risk of baro/volutrauma and oxygen toxicity (PIP and $\mathrm{FIO}_{2}$ ) and the consequent reduction in MV aggressiveness. Razavi et al. (34), assessing iNO effects on pulmonary inflammation in a mouse model of sepsis-induced acute lung injury, demonstrated that early iNO exposure was associated with reduced pulmonary leukocyte infiltration and less oxidative injury. These authors concluded that early iNO administration is of clinical benefit in the natural history of acute lung injury in clinical settings. Moreover, it seems that iNO response is better in patients with more severe respiratory failure, as reported by Dobyns et al. (16). As our historical group was statisti- 
Table 5. Ventilator settings and gas exchange at enrollment in the conventional therapy group (CTG) and in the inhaled nitric oxide group (iNOG)

\begin{tabular}{|c|c|c|c|}
\hline & CTG $(\mathrm{n}=21)$ & iNOG $(\mathrm{n}=18)$ & $p$ Value \\
\hline$P_{a w}$ & $16.90 \pm 4.5$ & $19.48 \pm 4.4$ & $>.05$ \\
\hline PEEP, $\mathrm{cm} \mathrm{H}_{2} \mathrm{O}$ & $11.87 \pm 1.28$ & $12.31 \pm 1.58$ & $>.05$ \\
\hline $\mathrm{PaCO}_{2}$, torr & $40.40(30.3-64.3)$ & $55.15(20.7-92.2)$ & $>.05$ \\
\hline $\mathrm{PaO}_{2} / \mathrm{FIO}_{2}$ & $116.89 \pm 34.5$ & $62.49 \pm 12.8$ & $<.0001$ \\
\hline $\mathrm{OI}$, torr/cm H $\mathrm{H}_{2} \mathrm{O}$ & $15.2(7.2-32.2)$ & $24.3(16.3-70.4)$ & $<.0001$ \\
\hline
\end{tabular}

$\mathrm{P}_{\mathrm{aw}}$, mean airway pressure; PEEP, positive end-expiratory pressure; OI, oxygenation index (mean airway pressure $\times \mathrm{FIO}_{2} \times 100 / \mathrm{PaO}_{2}$ ).

Tests: $t$-test and Mann-Whitney. Values are mean $\pm \mathrm{SD}$; median (range).

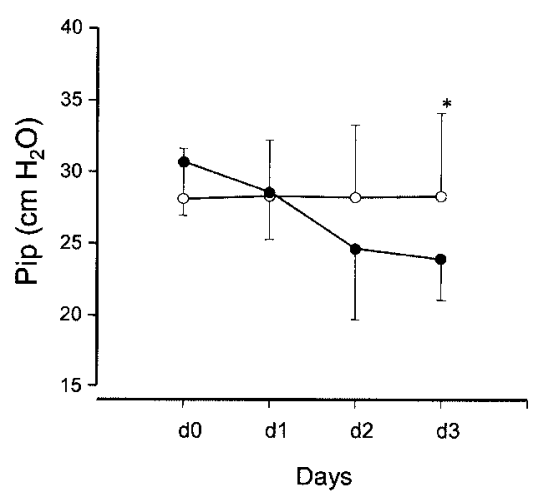

A

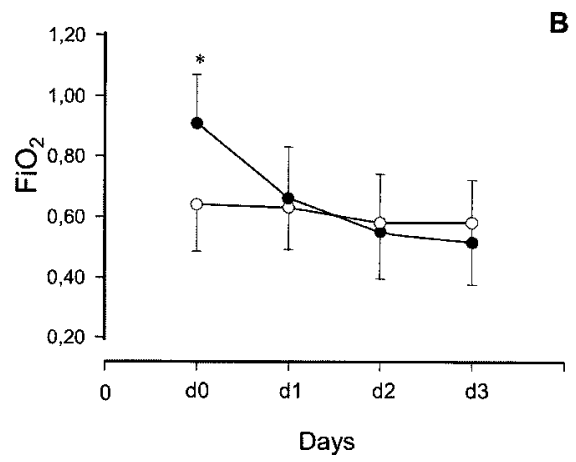

Figure 2. $A$, evolution of means of peak inspiratory pressure (Pip, $\mathrm{cm} \mathrm{H}_{2} \mathrm{O}$ ); and $\mathrm{B}$, $\mathrm{FIO}_{2}$ over the treatment period $(d 0-d 3)$ for both groups. The conventional therapy group values are represented as open circles and the inhaled nitric oxide group values as filled circles. ${ }^{*} p<.05$, Friedman test.

cally comparable with iNOG, and iNOG lung function was even worse than CTG, we believe that our findings may be explained by the early iNO administration.

Many studies have given no value to iNO therapy because of a lack of impact on mortality rate $(9,10,15,16,33)$. However, it should be considered that the improvement in oxygenation promoted by $\mathrm{NO}$ inhalation therapy may contribute to decreased MV intensity, as we have demonstrated. This, in turn, may reduce ventilator-induced lung injury, facilitate the use of protective ventilatory strategies, and have a positive effect on mortality rate. This hypothesis needs to be tested in larger randomized controlled trials.

Lundin et al. (35) was the only study in The Cochrane Library review (22) to describe the influence of iNO therapy on the length of stay in intensive care. These authors found no difference in intensive care stay when using iNO therapy; this is in agreement with our findings. We also found no difference in MV duration. Because of the small number of iNOG patients who died in our study, it is difficult to make any further conclusions on these issues at this time.

Inhaled NO Weaning, Side Effects, and Toxicity. The abrupt withdrawal of NO inhalation has been shown to produce severe pulmonary vasoconstriction, known as the "rebound" phenomenon $(6$, 36). Two of our children presented "rebound" and we had to increase $\mathrm{FIO}_{2}$ and restart iNO during the weaning process.

Inhaled NO toxicity is mainly related to the formation of $\mathrm{NO}_{2}$ and methemoglobin. Nitrogen dioxide production rate depends on iNO dose, $\mathrm{FiO}_{2}$, and length of iNO treatment, the amount of $\mathrm{NO}_{2}$ formed being $1.14 \%$ of the NO dose (37). Administration of the lowest iNO dose for the shortest period in our protocol did not increase $\mathrm{NO}_{2}$ levels $>1.5 \mathrm{ppm}$. This agrees with other studies performed in children $(12,14-16,38)$.

The reaction of NO with hemoglobin produces methemoglobin. A methemoglobin concentration $>2 \%$ of total hemoglobin can impair oxygen unloading and worsen tissue hypoxia. Far higher doses of iNO than those clinically used are not expected to cause significant methemoglobinemia in adults (8). Only two reports have been published on significant methemoglobinemia during iNO therapy in neonates $(39,40)$. We did not observe methemoglobin concentrations $>1 \%$ to-

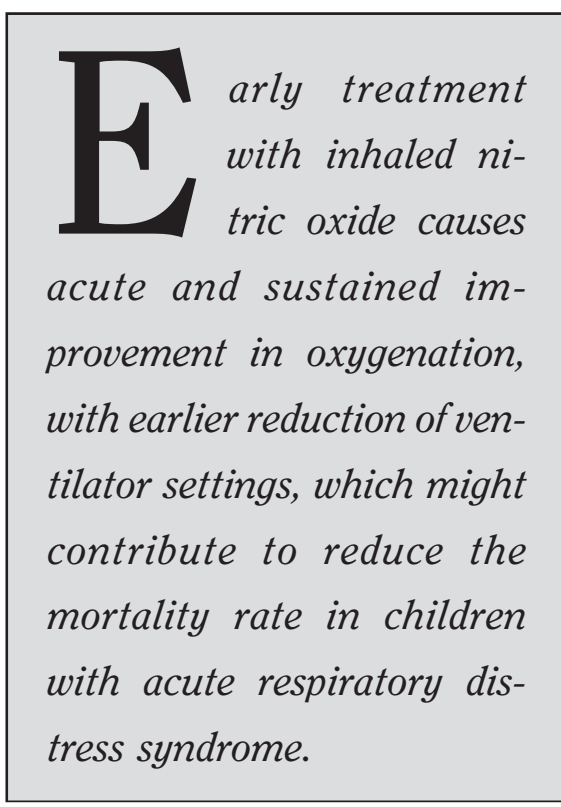

tal hemoglobin as seen in other studies with children $(10-12,14,15,20,38)$. This suggests that iNO is safe for use with children in low doses and with careful monitoring.

Study Limitations. The statistical power and level of evidence in this study are limited due to its observational and unrandomized design and the small number of patients. Despite patients having received the same standard ARDS protocol (fluid therapy, catecholamines, prone positioning, MV protective strategy, and PEEP levels), cointerventions over the 4-yr study period, as well as PICU staff learning curve, could have varied and contributed to the outcome.

\section{CONCLUSIONS}

Early treatment with iNO causes acute and sustained improvement in oxygenation, with earlier reduction in ventilator settings, which might contribute to reduce mortality rate in children with ARDS. Length of stay in intensive care and duration of mechanical ventilation are not changed despite the iNO group being sicker than the control group.

In view of the complexity of ARDS pathophysiology, it will be very difficult to find a single therapy for the management of this syndrome. Recognizing the limitations of this study due to number of cases and retrospective controls, we suggest that early and/or sustained administration of iNO should be subjected to randomized controlled clinical trials in acute lung injury in the PICU setting. 


\section{ACKNOWLEDGMENTS}

We thank the entire PICU staff for expert assistance and Colin E. Knaggs for text revision.

\section{REFERENCES}

1. Ashbaugh DG, Bigelow DB, Petty TL, et al: Acute respiratory distress in adults. Lancet 1967; 12:319-322

2. McIntyre RC Jr, Pulido EJ, Bensard DD, et al: Thirty years of clinical trials in acute respiratory distress syndrome. Crit Care Med 2000; 28:3314-3331

3. Rossaint R, Falke KJ, Lopez FA, et al: Inhaled nitric oxide for adult respiratory distress syndrome. $N$ Engl J Med 1993; 328:399-405

4. Abman SH, Griebel JL, Parker DK, et al: Acute effects of inhaled nitric oxide in children with severe hypoxemic respiratory failure. J Pediatr 1994; 124:881-888

5. Johanningman DA, Davis K Jr, Campbell RS, et al: Inhaled nitric oxide in acute respiratory distress syndrome. J Trauma 1997; 43: 904-910

6. Manktelow C, Bigatello LM, Hess D, et al: Physiologic determinants of the response to inhaled nitric oxide in patients with acute respiratory distress syndrome. Anesthesiology 1997; 82:297-307

7. Michael JR, Barton RG, Saffle JR, et al: Inhaled nitric oxide versus conventional therapy. Effects on oxygenation in ARDS. Am J Respir Crit Care Med 1998; 157:1372-1380

8. Lotti GA, Olivei MC, Palo A, et al: Acute effects of inhaled nitric oxide in adult respiratory distress syndrome. Eur Respir J 1998; 12:1164-1171

9. Troncy E, Collet JP, Shapiro S, et al: Inhaled nitric oxide in acute respiratory distress syndrome. A pilot randomized controlled study. Am J Respir Crit Care Med 1998; 157: 1483-1488

10. Day RW, Guarin M, Lynch JM, et al: Inhaled nitric oxide in children with severe lung disease: Results of acute and prolonged therapy with two concentrations. Crit Care Med 1996; 24:215-221

11. Nakagawa TA, Morris A, Gomez RJ, et al: Dose response to inhaled nitric oxide in pediatric patients with pulmonary hypertension and acute respiratory distress syndrome. J Pediatr 1997; 131:63-69

12. Goldman AP, Tasker RC, Hosiasson S, et al: Early response of inhaled nitric oxide and its relationships to outcome in children with severe hypoxemic respiratory failure. Chest 1997; 112:752-758

13. Day RW, Allen EM, Witte MK: A randomized, controlled study of the 1-hour and 24-hour effects of inhaled nitric oxide therapy in chil- dren with acute hypoxemic respiratory failure. Chest 1997; 112:1324-1331

14. Okamoto K, Hamaguchi M, Kukita I, et al: Efficacy of inhaled nitric oxide in children with ARDS. Chest 1998; 114:827-833

15. Ream RS, Hauver JF, Lynch RE, et al: Lowdose inhaled nitric oxide improves the oxygenation and ventilation of infants and children with acute, hypoxemic respiratory failure. Crit Care Med 1999; 27:989-996

16. Dobyns EL, Cornfield DN, Anas NG, et al: Multicenter randomized controlled trial of the effects of inhaled nitric oxide therapy on gas exchange in children with acute hypoxemic respiratory failure. J Pediatr 1999; 134: 406-412

17. Lowson SM, Rich GF, McArdle PA, et al: The response to varying concentrations of inhaled nitric oxide in patients with acute respiratory distress syndrome. Anesth Analg 1996; 82:574-581

18. Clark RH: How do we safely use inhaled nitric oxide? Pediatrics 1999; 103:296-297

19. Friese RS, Fullerton DA, McIntyre RC, et al: NO prevents neutrophil-mediated pulmonary vasomotor dysfunction in acute lung injury. J Surg Res 1996; 63:23-28

20. Demirakça S, Dotsch J, Knothe C, et al: Inhaled nitric oxide in neonatal and pediatric acute respiratory distress syndrome: Dose response, prolonged inhalation, and weaning. Crit Care Med 1996; 24:1913-1919

21. Fioretto JR, Bonatto RC, Ricchetti SMQ, et al: Early administration of inhaled nitric oxide to children with acute respiratory distress syndrome and its effects on oxygenation and ventilator settings: Prospective preliminary report of ten patients. Croat Med J 2001; 42:527-534

22. Sokol J, Jacobs SE, Bohn D: Inhaled nitric oxide for acute hypoxemic respiratory failure in children and adults (Cochrane Review). The Cochrane Library 2002; 4

23. Bernard GR, Artigas A, Brigham KL, et al: Report of the American-European Consensus Conference on ARDS: Definitions, mechanisms, relevant outcomes and clinical trial coordination. Intensive Care Med 1994; 20: 225-232

24. Fioretto JR, Ferrari GF, Ricchetti SMQ, et al: Síndrome do Desconforto Respiratório Agudo em Crianças: Incidência, Mortalidade e Trocas Gasosas. Rev Bras Terap Intens 2001; 2:58-62

25. Pollack MM, Patel KM, Ruttimann UE: PRISM III: An update Pediatric Risk of Mortality score. Crit Care Med 1996; 24:743-752

26. Wilkinson JD, Pollack MM, Glass NL, et al: Mortality with multiple organ system failure and sepsis in pediatric intensive care unit. J Pediatr 1987; 111:324-328

27. Members of the American College of Chest Physicians/Society for Critical Care Medicine
Consensus Conference Committee: Definitions for sepsis and organ failure and guidelines for the use of innovative therapies in sepsis. Crit Care Med 1992; 20:864-874

28. Numa AH, Hammer J, Newth CJL: Effect of prone and supine positions on functional residual capacity, oxygenation, and respiratory mechanics in ventilated infants and children. Am J Respir Crit Care Med 1997; 1156: $1185-1189$

29. Cuthbertson BH, Dellinger P, Dyar OJ, et al: UK guidelines for the use of inhaled nitric oxide therapy in adult ICUs. Intensive Care Med 1997; 23:1212-1218

30. Francoe M, Troncy E, Blaise G: Inhaled nitric oxide: Technical aspects of administration and monitoring. Crit Care Med 1998; 26 : 782-796

31. Finer NN, Etcher PC, Kamstra B, et al: Inhaled nitric oxide in infants refereed to extracorporeal membrane oxygenation: Dose response. J Pediatr 1994; 124:302-308

32. Lonnqvist PA: Inhaled nitric oxide in newborn and pediatric patients with pulmonary hypertension and moderate to severe impaired oxygenation: Effects of doses of 3-100 parts per million. Intensive Care Med 1997; 23:773-779

33. Dellinger RP, Zimmerman JL, Taylor RW, et al: Effects of inhaled nitric oxide in patients with acute respiratory distress syndrome: Results of a randomized phase II trial. Crit Care Med 1998; 26:15-23

34. Razavi HM, Werhun R, Scott JA, et al: Effects of inhaled nitric oxide in a mouse model of sepsis-induced acute lung injury. Crit Care Med 2002; 30:868-873

35. Lundin S, Mang $\mathrm{H}$, Smithies M, et al: Inhalation of nitric oxide in acute lung injury: Results of a European multicentre study. Intensive Care Med 1999; 25:911-919

36. Troncy E, Francoeur M, Blaise G: Inhaled nitric oxide: Clinical applications, indications, and toxicity. Can J Anaesth 1997; 44: 973-988

37. Breuer J, Waidelich F, Von Brenndorff I, et al: Technical considerations for inhaled nitric oxide therapy: Time response to nitric oxide dosing changes and formation of nitric dioxide. Intensive Care Med 1997; 156: 460-462

38. Tang SF, Sherwood MC, Miller OI: Randomized trial of three doses of inhaled nitric oxide in acute respiratory distress syndrome. Arch Dis Child 1998; 79:415-418

39. Heal CA, Spencer SA: Methaemoglobinemia with high-dose nitric oxide administration. Acta Paediatr 1995; 84:1318-1319

40. Frostell CG, Lonngvist PA, Sonesson SE, et al: Near fatal pulmonary hypertension after surgery repair of congenital diaphragmatic hernia. Successful use of inhaled nitric oxide. Anaesthesia 1993; 48:679-683 